\title{
An Fuzzy Neural Approach for Medical Image Retrieval
}

\author{
${ }^{1}$ C. Sriramakrishnan and ${ }^{2}$ A. Shanmugam \\ ${ }^{1}$ Department of ECE, Adhiyamaan College of Enginering, Hosur, India \\ ${ }^{2}$ Bannari Amman Institute of Technology, Sathy, Tamil Nadu, India
}

Received 2012-07-30, Revised 2012-08-21; Accepted 2012-09-05

\begin{abstract}
Image retrieval based on a query image is necessary for effective and efficient use the information that is stored in medical image databases. Medical Image Retrieval is difficult as not only the localization and directionality of human visual system is to be considered but also the pathological condition. Image identification and segmentation for feature extraction pose a challenge to image retrieval process. Challenges posed include large number of images to be processed for the image retrieval and identifying the region of interest automatically to optimize the search. In this study, we propose a novel image segmentation algorithm Fuzzy Edge Detection and Segmentation (FEDS). The proposed FEDS algorithm is tested on medical images and for classification of images, a bell fuzzy multilayer perceptron is proposed. The proposed neural network Bell Fuzzy Multi Layer Perceptron (BF-MLP) Neural network is constructed by introducing a fuzzy logic in hidden layer with the sugeno model and bell function. The proposed neural network consists of two layers with the first layer being a tanh activation function and the second layer containing the bell fuzzy activation function. The proposed FEDS method was implemented using Matlab and Modelsim. A total of 44 images were considered with three class labels. The edge obtained for which segmentation is done using the proposed segmentation algorithm. The proposed BF-MLP neural network algorithm was implemented using Visual Studio and the classification accuracy compared with MLP Neural Network with sigmoid activation function. In this study, a fuzzy segmentation algorithm and a fuzzy classification algorithm is proposed to improve the medical image retrieval accuracy. The proposed segmentation algorithm, Fuzzy Edge Detection and Segmentation (FEDS), was implemented using Matlab and features were extracted using Fast Hartley Transform (FHT). The extracted features were used to train the proposed neural network, Bell Fuzzy Multi Layer Perceptron Neural Network (BF-MLP). 44 images with 3 class labels were used to test the algorithm and classification accuracy of $93.2 \%$ was obtained.
\end{abstract}

Keywords: Content Based Image Retrieval (CBIR), Medical Image, Fuzzy Edge Detection and Segmentation (FEDS), Bell Fuzzy Multilayer Perceptron

\section{INTRODUCTION}

With the availability of large amount of digital medical images in databases, image retrieval based on a query image is necessary for effective and efficient use of the information available. Content Based Image Retrieval (CBIR) is the process of locating similar picture/pictures in an image database when a query image is provided. When the picture of a car is given, the system should retrieve similar car images in the database. Automated extraction of features from the image is crucial for image retrieval systems (Rui et al., 1999). This is achieved through extracting image features like color, texture and shape. Such image features are then compared to the query image and database images. A similarity algorithm calculates the similarity degree between both images. Database images which have same features as the query image (with the highest similarity measure) are then ranked and presented to the user.

CBIR is a two step process which includes Feature Extraction and Image Matching (also called feature Corresponding Author: Sriramakrishnan, C., Department of ECE, Adhiyamaan College of Enginering, Hosur, India Tel: 91+9443774452 
matching). Feature Extraction extracts image features to a discerning extent and information like color, texture and shape extracted from images are called feature vectors. Extraction is undertaken on query and database images. Image matching includes use of features of both images and comparison between them to ensure features similar to those in database images.

Image segmentation represents a digital image in simple multiple region forms to facilitate analysis. Objects, boundaries in an image are sited and also object and background is segmented using image segmentation (Gonzalex and Woods, 2009; Banerjee and Kundu, 2003; Amartur et al., 1992; Lo et al., 1995; Hussain et al., 2012; Barskar and Ahmed, 2011). Generally, image segmentation process first segments color which is followed by edge detection. Feature extraction depends upon the quality of segmentation as over segmentation leads to many details of the object and under segmentation groups many object into single region. So level of segmentation required is a deciding factor for success or failure of analysis.

The image segmentation algorithm takes advantage of discontinuity and similarity of the image grey levels. Some of the most commonly used segmentation techniques are histogram thresholding, clustering, vector based and fuzzy technique.

In this study, a novel image segmentation algorithm Fuzzy Edge Detection and Segmentation (FEDS) is proposed. The proposed FEDS algorithm is tested on medical images and for classification of images, a bell fuzzy multilayer perceptron is proposed. The rest of the study is organized as follows: Section 2 reviews fuzzy techniques used in CBIR available in literature, section 3 details about the proposed methods, section 4 gives the experimental details and results and section 5 concludes the study.

\section{MATERIALS AND METHODS}

The dataset consists of 44 medical images from the National Biomedical Imaging archive for the experimentation of image retrieval. Images were segmented using the proposed Fuzzy Edge Detection Segmentation (FEDS) algorithm. Features were extracted from the segmented images using Fast Hartley Transform. A bell fuzzy multilayer perceptron is proposed for image classification.

\subsection{Fuzzy Edge Detection and Segmentation (FEDS)}

Edge detection is a process of identifying and locating edges in image for image segmentation. Distortion, noise, overlaps, intensity variation are some of the factors which contributes to edge extraction (Carron and Lambert, 1995). There are many methods in literature for edge detection; fuzzy method is used in this study.

Fuzzy theory is effective for modeling ambiguity or uncertainty, thus, the noise is efficiently removed from the image when detecting edges. The image is transformed into fuzzy image by the use of fuzzification function (Rey-Sern, 2008). In fuzzy segmentation, the regions in the image are obtained by connecting and merging the constant color inside image. Propinquity, a metric for closeness between components, defined by the fuzzy inference system is used. The propinquity measure depends on the color distance and the topological relation between components. The fuzzy segmentation method constructs clearer segmentation.

In the proposed algorithm, fuzzy logic is used to find the edge based on the output of the two edge detection method. Initially the edge is detected using Sobel operator. The Sobel operator filter kernel is used and is given by Eq. 1 and 2:

$$
\begin{aligned}
& \mathrm{H}_{\mathrm{x}}=\left[\begin{array}{ccr}
-1 & 0 & 1 \\
-2 & 0 & 2 \\
-1 & 0 & 1
\end{array}\right] \\
& \mathrm{H}_{\mathrm{y}}=\left[\begin{array}{crr}
1 & 2 & 1 \\
0 & 0 & 0 \\
-1 & 0 & -1
\end{array}\right]
\end{aligned}
$$

The vertical and horizontal gradient component $H_{x}$ and $\mathrm{H}_{\mathrm{y}}$ are obtained by the above kernels. The gradient magnitude is approximated as follows Eq. 3:

$$
\mathrm{H}=\left|\mathrm{H}_{\mathrm{x}}\right|+\left|\mathrm{H}_{\mathrm{y}}\right|
$$

And the angle of the orientation of the edge relative to the pixel grid is given by Eq. 4 :

$$
\varphi=\arctan \left(\frac{\mathrm{H}_{\mathrm{x}}}{\mathrm{H}_{\mathrm{y}}}\right)
$$

The two kernels can be simultaneously applied on image to obtain the approximate magnitude as follows Eq. 5 and 6:

$$
\mathrm{H}=\left[\begin{array}{ccc}
\mathrm{a}_{1} & \mathrm{a}_{2} & \mathrm{a}_{3} \\
\mathrm{a}_{4} & \mathrm{a}_{5} & \mathrm{a}_{6} \\
\mathrm{a}_{7} & \mathrm{a}_{8} & \mathrm{a}_{9}
\end{array}\right]
$$




$$
|H|=\left|\begin{array}{l}
\left(a_{1}+2 a_{2}+a_{3}\right)-\left(a_{7}+2 a_{8}+a_{9}\right) \\
+\left(a_{3}+2 a_{6}+a_{9}\right)-\left(a_{1}+2 a_{4}+a_{7}\right)
\end{array}\right|
$$

In the second method the Laplacian operator kernel given by Eq. 7 is used:

$$
\mathrm{I}=\left[\begin{array}{lrr}
0 & -1 & 0 \\
-1 & 4 & -1 \\
0 & -1 & 0
\end{array}\right]
$$

The magnitude of the image is given by Eq. 8:

$$
|I|=\left|\left(4 a_{5}-\left(a_{2}+a_{4}+a_{6}+a_{8}\right)\right)\right|
$$

The magnitudes from both the methods are normalized to obtain values in the range $[0,1]$. The normalized outputs are mapped to three class values $\mathrm{V}_{1}, \mathrm{~V}_{\mathrm{m}}$ and $\mathrm{V}_{\mathrm{h}}$ from Fig. 2 the range of the class values are given by:

$$
\begin{aligned}
& \mathrm{V}_{\mathrm{t}}=[0,0.8] \\
& \mathrm{V}_{\mathrm{m}}=[0,0.8] \\
& \mathrm{V}_{\mathrm{h}}=[0.8,1]
\end{aligned}
$$

The output of the fuzzy system determines whether the pixel is an edge or not based on the defined fuzzy rules. The three classes of outputs are $E_{l}, E_{m}$ and $E_{h}$ which defines the probability of the pixel being an edge is low, medium or high.

If $\mathrm{p}_{1}$ and $\mathrm{p}_{2}$ are the corresponding values from the two edge detection methods, some of the fuzzy rules can be described by:

- If $\mathrm{p}_{1}$ in $\mathrm{V}_{1}$ and $\mathrm{p}_{2}$ in $\mathrm{V}_{\mathrm{k}}$ then the probability of edge is classified to $\mathrm{E}_{\mathrm{m}}$

- If $\mathrm{p}_{1}$ in $\mathrm{V}_{\mathrm{h}}$ and $\mathrm{p}_{2}$ in $\mathrm{V}_{\mathrm{h}}$ then the probability of edge is classified to $E_{h}$

A total of 9 combinations are possible with generation of 9 rules. The outer edge value is used to create a mask and features are extracted using Fast Hartley Transform.

The Hartley transforms maps real-valued temporal or spacial functions into real-valued frequency function decomposing into two independent sets of sinusoidal components (Hartley, 1942). Energy features are extracted using the Fast Hartley Transform on the extracted segments. The obtained energies are used to train the neural network.

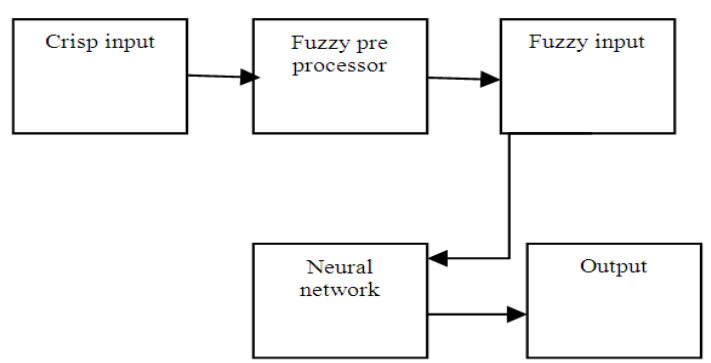

Fig. 1. Block diagram showing preprocessing fuzzy logic to neural network

Table 1. Design metrics of the proposed neural network model

\begin{tabular}{ll}
\hline Input Neuron & 20 \\
\hline Output Neuron & 1 \\
Number of Hidden Layer & 2 \\
Number of processing elements-first layer & 6 \\
Transfer function of first hidden layer & tanh \\
Learning rule & momentum \\
Number of processing elements-second layer & 2 \\
Transfer function of second hidden layer & bellfuzzy \\
Learning Rule of hidden layer & Momentum \\
\hline
\end{tabular}

\subsection{Multilayer Perceptron Neural Network}

Multilayer Perceptrons (MLPs) are feed forward neural networks; these are supervised networks and widely used for modeling prediction problems (Hornik et al., 1989). During training the network learns how to transform input data into a desired response, the MLPs are generally trained with the standard back propagation algorithm. Back propagation computes the sensitivity of the output with respect to each weight in the network and modifies each twilight by a value that is proportional to the sensitivity. In training process, the representative examples are iteratively presented to the network, so that it can integrate this knowledge within its structure. A good training algorithm not only shortens the training time but also achieves better accuracy.

There are a number of ways fuzzy logic can be used with neural networks. The fuzzy logic is used in the form of a fuzzifier function to preprocess or post-process data for a neural network as shown in Fig. 1. In the second method fuzziness can enter neural networks to define the weights from fuzzy sets.

\subsection{Proposed Neural Network Model \\ 3.2. Bell Fuzzy Multi Layer Perceptron Neural Network (BF-MLP Neural Network)}

The proposed neural network Bell Fuzzy Multi Layer Perceptron (BF-MLP) Neural network is constructed by introducing a fuzzy logic in hidden layer with the sugeno model and bell function. The proposed neural network 
consists of two layers with the first layer being a tanh activation function and the second layer containing the bell fuzzy activation function.

The Bell Fuzzy Multi Layer Perceptron (BF-MLP) Neural network proposed in this study uses the criteria specified in Table 1.

The Sugeno fuzzy model is the fuzzy model introduced in the proposed algorithm. A typical fuzzy rule in Sugeno fuzzy model has the form If $x$ is $A$ and $y$ is $B$ then $z=f(x, y)$. where A and B are fuzzy sets in the antecedent part, while $\mathrm{z}=\mathrm{f}(\mathrm{x}, \mathrm{y})$ is crisp function in the consequent part (Takagi and Sugeno, 1985).

The fuzzy bell membership function is given by:

$$
\frac{1}{1+\left|\frac{\mathrm{x}-\mathrm{w}_{2}}{\mathrm{w}_{0}}\right|^{2 \mathrm{wi}}}
$$

where, $\mathrm{x}$ is the input and $\mathrm{w}_{\mathrm{i}}$ is the weight.

\section{RESULTS AND DISCUSSION}

The proposed FEDS method was implemented using Matlab and Modelsim. A total of 44 images were considered with three class labels. Images used in the experimental setup are shown in Fig. 2 and 3 shows the edge obtained for which segmentation is done using the proposed segmentation algorithm.
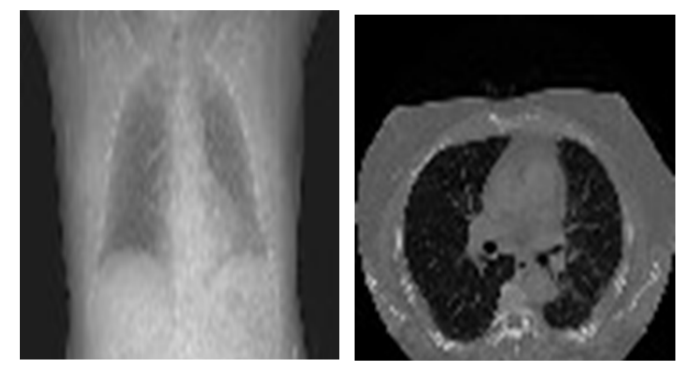

Fig. 2. Images used in the experiment
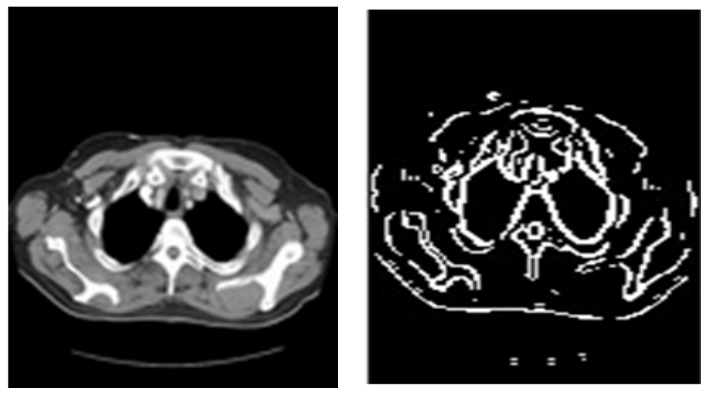

Fig. 3. Input image with obtained edges from proposed method
The proposed BF-MLP neural network algorithm was implemented using Visual Studio and the classification accuracy compared with MLP Neural Network with sigmoid activation function.

Results of the classification accuracy obtained are displayed in Fig. 4 and 2. Table 2 and 3 tabulates the precision, recall and $\mathrm{f}$ Measure of the various images used in the experiment. Figure 5 and 6 shows the precision, recall and $\mathrm{f}$ Measure of the various images used in the experiment.

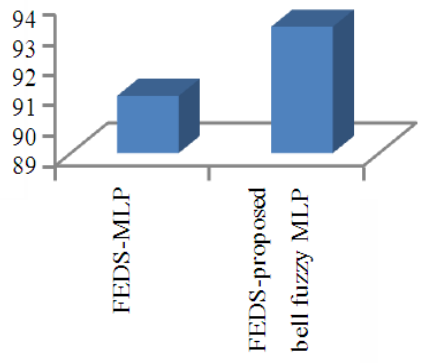

Classification accuracty

Fig. 4. Classification accuracy obtained

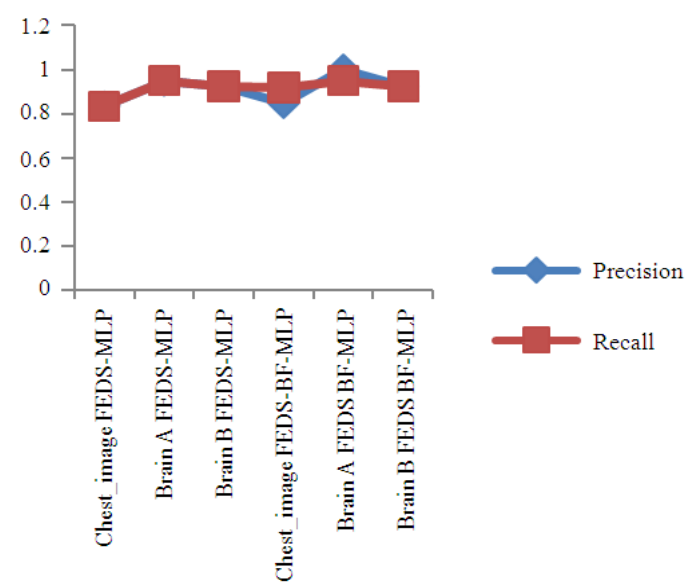

Fig. 5. Precision and Recall

Table 2. Classification Accuracy

\begin{tabular}{ll}
\hline Technique used & $\begin{array}{l}\text { Classification } \\
\text { accuracy } \%\end{array}$ \\
\hline FEDS-MLP & 90.9 \\
FEDS-proposed bell fuzzy MLP & 93.2 \\
\hline
\end{tabular}




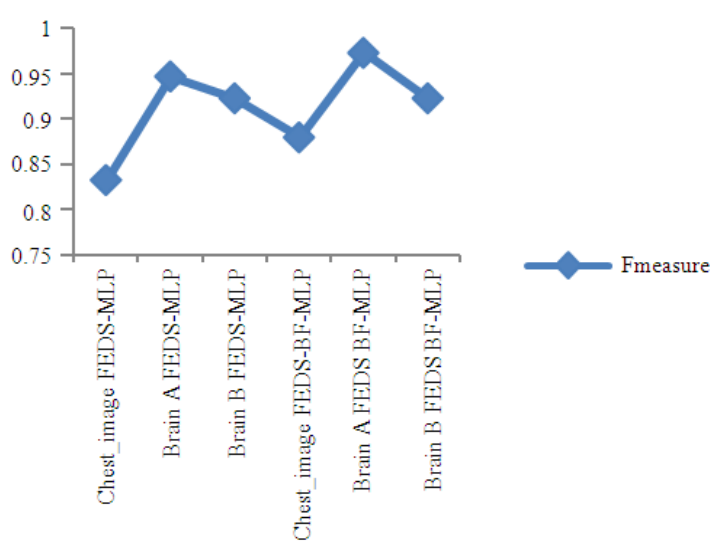

Fig. 6. f Measure

Table 3. Summary of proposed fuzzy classifier and FEDS

\begin{tabular}{lllllll}
\hline $\begin{array}{l}\text { Classifi- } \\
\text { Cation }\end{array}$ & FEDS-MLP & & FEDS-proposed bell fuzzy MLP \\
Accuracy & $90.90 \%$ & & & $93.20 \%$ & \\
\hline $\begin{array}{l}\text { Class } \\
\text { chest_ }\end{array}$ & $\begin{array}{l}\text { Precision } \\
\text { image }\end{array}$ & Recall & Fmeasure & Precision & Recall & Fmeasure \\
BrainA & 0.947 & 0.833 & 0.833 & 0.846 & 0.917 & 0.880 \\
BrainB & 0.923 & 0.947 & 0.947 & 1.000 & 0.947 & 0.973 \\
\hline
\end{tabular}

\section{CONCLUSION}

In this study, a fuzzy segmentation algorithm and a fuzzy classification algorithm is proposed to improve the medical image retrieval accuracy. The proposed segmentation algorithm, Fuzzy Edge Detection and Segmentation (FEDS), was implemented using Matlab and features were extracted using Fast Hartley Transform (FHT). The extracted features were used to train the proposed neural network, Bell Fuzzy Multi Layer Perceptron Neural Network (BF-MLP). 44 images with 3 class labels were used to test the algorithm and classification accuracy of $93.2 \%$ was obtained.

\section{ACKNOWLEDGEMENT}

The authors are extremely thankful to Computational Visual Cognition Laboratory and websites mentioned in the references.

\section{REFERENCES}

Amartur, S.C., D. Piraino and Y. Takefuji, 1992. Optimization neural networks for the segmentation of magnetic resonance images. IEEE Trans. Med. Imag., 11: 215-220. PMID: 18218375
Banerjee, M. and M.K. Kundu, 2003. Content based image retrieval with fuzzy geometrical features. Proceedings of the 12th IEEE International Conference on Fuzzy Systems, May 25-28, IEEE Xplore Press, pp: 932-937. DOI: 10.1109/FUZZ.2003.1206556

Barskar, R. and G.F. Ahmed, 2011. CBIR using fuzzy edge detection mask. Proceeding of the 2011 International Conference on Communication, Computing and Security, (ICCCS' 11), ACM New York, USA., pp: 277-280. DOI: 10.1145/1947940.1947999

Carron, T. and P. Lambert, 1995. Fuzzy color edge extraction by inference rules quantitative study and evaluation of performances. Proceedings of the International Conference on Image Processing, (ICIP' 95), ACM Press, USA., pp. 2181-2181.

Gonzalex, R.C. and R.E. Woods, 2009. Digital Image Processing. 3rd Edn., Pearson Education India, India, ISBN-10: 8131726959, pp: 954.

Hartley, R.V.L., 1942. A more symmetrical fourier analysis applied to transmission problems. Proc. IRE, 30 : 144-150. DOI: 10.1109/JRPROC.1942.234333

Hornik, K., M. Stinchcombe and H. White, 1989. Multilayer feedforward networks are universal approximators. Neural Netw., 2: 359-366. DOI: 10.1016/0893-6080(89)90020-8

Hussain, S.J., T.S. Savithri and P.V.S. Devi, 2012. Segmentation of tissues in brain MRI images using dynamic neuro-fuzzy technique. Int. J. Soft Comput. Eng., 1: 416-423.

Lo, S.C.B., S.L.A. Lou, J.S. Lin, M.T. Freedman and M.V. Chien et al., 1995. Artificial convolution neural network techniques and applications for lung nodule detection. IEEE Trans. Med. Imag., 14: 711718. DOI: $10.1109 / 42.476112$

Rey-Sern, L., 2008. Edge detection by morphological operations and fuzzy reasoning. Proceedings of the 2008 Congress on Image and Signal Processing, (CISP' 08), ACM Press, USA., pp: 729-733. DOI: 10.1109/CISP.2008.346

Rui, Y., T.S. Huang and S.F. Chang, 1999. Image retrieval: Current techniques, promising directions and open issues. J. Vis. Commun. Image Represent., 10: 39-62.

Takagi, T. and M. Sugeno, 1985. Fuzzy identification of systems and its applications to modeling and control. IEEE Trans. Syst. Man Cybern., 15: 116-132. 